\title{
Investigating the risk factors for antibiotic lock therapy failure in pediatric cancer: a single center retrospective analysis
}

\author{
Hatice Karaoğlu Asrak ${ }^{1 \oplus}$, Nurşen Belet ${ }^{1 \oplus}$, Özlem Tüfekçi ${ }^{2 \oplus}$, Canan Özlü $^{1 \oplus}$, \\ Birsen Baysal ${ }^{2 \oplus}$, Dilek İnce ${ }^{2 \oplus}$ \\ Divisions of ${ }^{1}$ Pediatric Infectious Diseases, ${ }^{2}$ Pediatric Hematology and Oncology, Department of Pediatrics, Dokuz Eylul University \\ Faculty of Medicine, İzmir, Turkey.
}

\begin{abstract}
Background. Catheter-related bloodstream infection (CRBSI) is one of the most common complications of central lines. Data concerning the effectiveness and safety of antibiotic lock therapy (ALT), especially in pediatric hematology and oncology patients, have not yet reached sufficient levels of evidence. We aimed to share our center's experience on ALT in pediatric cancer and to investigate the causes of ALT failure.
\end{abstract}

Methods. All cases with CRBSI and treated with ALT administiration in children with cancer between January 2015 and May 2019 were reviewed. Patients characteristics, laboratory and clinical findings, treatments, outcome of ALT, recurrences and reinfections were recorded. Patients with successful and unsuccessful ALT outcomes were compared in order to identify the risk factors for ALT failure.

Results. Sixteen eligible CRBSI treated with adjunctive ALT were identified. The most common pathogens were coagulase negative staphylococci $(8 / 16,50 \%)$. Treatment failure was observed in $31.2 \%(5 / 16)$. Younger age alone was an independent risk factor for treatment failure ( 0.9 vs 6.8 years, $p=0.038)$. Recurrence and reinfection rates were $23.1 \%$ and $16.7 \%$. Mild bleeding occured in two cases (12.5\%) and occlusion causing catheter removal was seen in one $(6.3 \%)$.

Conclusions. ALT was found to be a safe modality with a success rate of $68.8 \%$ in children with cancer at our center and younger age was an independent risk factor for treatment failure. Future studies with larger sample sizes are needed to determine the factors affecting the ALT outcome, especially in childhood malignancies.

Key words: antibiotic lock therapy, catheter-related bloodstream infections, cancer, childhood malignancy.

Central venous catheters (CVCs) are critical for children with cancer owing to difficulties in intravenous access, the necessity of safe administration of chemotherapeutics, frequent need for treatments such as parenteral nutrition, blood products and antibiotics. Catheterrelated bloodstream infections (CRBSI) are the most common complication of central lines and can affect up to one-fourth of children with cancer during their treatment course. ${ }^{1-3}$ Besides

$凶$ Hatice Karaoğlu Asrak

hatice_karaoglu@msn.com

Received 24th March 2020, revised 7th May 2020, accepted 21st May 2020. serious complications like endocarditis, septic thrombophlebitis and metastatic infection by hematogenous spread, CRBSI can also cause prolonged hospital stay, increased risk of mortality and hospital costs. ${ }^{2,4,5}$

When CRBSI is related to a long term CVC, catheter salvage can be attempted by administering both systemic antibiotics and antibiotic lock. The objective of antibiotic lock therapy (ALT) is to eliminate the microbial biofilm from long term CVC by locking a highconcentration antibiotic solution into CVCs. Although recommendations related to ALT seem clear, data concerning the effectiveness and safety of ALT -especially in children with 
cancer - have not yet reached sufficient levels of evidence. ${ }^{6,7}$

With this study we aimed to share our center's experience on ALT in pediatric cancer patients with long term catheters and to investigate the causes of treatment failure.

\section{Material and Methods}

\section{Patient selection}

Patients with cancer who have long-term central venous or port catheters, aged 0-18 years, received ALT for CRBSI from January 2015 to May 2019 at Dokuz Eylul University Hospital were enrolled. All data were collected retrospectively through patient files and medical records. The research was reviewed and approved by the Ethics Committee of Dokuz Eylul University Medical Faculty (No: 2020/04-53, date: 17.02.2020) and an informed consent was required for the participants.

\section{Definitions}

CRBSI was defined as isolation of a recognized pathogen (other than a common skin contaminant) from blood culture or of a common skin contaminant from at least two separate blood cultures in a patient with clinical findings and with no other source of infection other than the central catheter. Patients with only one blood culture positivity for common skin concomitants (Corynebacterium spp., Bacillus spp., Propionibacterium spp., coagulase-negative staphylococci, viridans group streptococci, Aerococcus spp. and Micrococcus spp...) or with another infection source or with a short-term central catheter were excluded from the study.

Recurrence was defined as occurrence of another CRBSI with the same organism and reinfection was defined as occurrence of another CRBSI with a different organism after both blood cultures from catheter hub and peripheral vein were cleared and clinical improvement was achieved. ${ }^{8,9}$
ALT success was defined as defervescence, clinical improvement and clearance of cultures in 72 hours and no recurrence or re-infection within 30 days after discontinuation of ALT.

ALT failure was considered in the presence of one of the following situations:

- Persistent bacteremia or persistence of clinical findings despite 72 hours of antibiotic therapy to which the infecting organism is susceptible or,

- Catheter removal due to infection, mortality potentially attributable to sepsis, recurrence or re-infection within 30 days after discontinuation of ALT.

\section{Data collection and follow-up period}

Data were collected retrospectively on participants' demographics, underlying diagnosis, inpatient unit, length of hospital stay until the CRBSI diagnosis, laboratory data, catheter type, date of catheter placement and removal, microbiologic data, total parenteral nutrition, antimicrobial treatment (systemic and lock therapies, duration, agent, concentration, anticoagulants) and anti-cancer therapies on a structured proforma.

A patient was included in the study only once with the first CRBSI episode treated with ALT. All patients were followed up for recurrence or re-infection until catheter removal, death or the end of the study period whichever came first.

Concerning the complications of ALT, medical records were also investigated for bleeding or occlusion of the catheter.

\section{Analysis}

Patients were divided into two groups according to ALT outcome and compared with each other in terms of age, gender, underlying disease, absolute neutrophil count (ANC), lymphocyte and monocyte count, CRP values, length of hospital stay until CRBSI, catheter day, catheter type, time to ALT, heparin concentration in the lock solution and dwell time. 
Statistical analysis was performed with the "IBM Statistical Social Sciences SPSS 22.0" software. Variables with nonnormal distribution were indicated with medians and interquartile range (IQR). The categorical data were analyzed using a chi-square test or the two-sided Fisher's exact test. The Mann-Whitney U test was used to test the nonnormal distribution of data. $\mathrm{P}$ values less than 0.05 were considered statistically significant.

\section{Results}

Between October 2015 and May 2019, 16 patients aged 0-18 years who had been followed up for childhood malignancies, had a permanent catheter and underwent ALT were included in the study. The median age was 3.8 (IQR: 0.911.5) years. Male to female ratio was $1.28 / 1$. Among nine hematologic malignancies (\% 56,3) only one was acute myeloblastic leukemia and the rest were acute lymphoblastic leukemia (ALL). The other diagnoses were neuroblastoma in four, brain tumor in two and hepatoblastoma in one patient (Table I).
Nine patients were receiving intensive chemotheraphy (induction phase for leukemias and active chemotheraphy for solid tumors); two patients with leukemia were in the consolidation phase and one was in reinduction phase of the treatment protocol. Two patients with leukemia were on immunosuppressive therapy in post-transplant period (matched sibling donor allogenic hematopoietic stem cell transplantation). Of the two remaining patients, one was receiving radiation therapy for her brain tumor, and the other had ALL and developed necrotizing pancreatitis.

The incidence of CRBSI was 5.64/1000 catheterdays among the cases included. The most prevalent pathogens identified in the study were coagulase negative staphylococci (CoNS) $(50 \%)$ and gram negative bacteria $(31.3 \%)$ followed by polymicrobial $(12.5 \%)$ and fungal $(6.2 \%)$ infections (Table II).

All patients recieved ALT and systemic antibiotics according to the sensitivity of the microorganism. Antibiotic concentrations and the duration of therapy were in accordance with

Table I. Demographic and clinical features of all 16 children.

\begin{tabular}{lc}
\hline Age [years (IQR)] & $3.8(0.9-11.5)$ \\
Gender [n (\%)] & $9(56.3)$ \\
$\quad$ Male & $7(43.8)$ \\
$\quad$ Female & $9(56.3)$ \\
Underlying diagnosis [n (\%)] & $7(43.8)$ \\
$\quad$ Leukemia & $2(12.5)$ \\
$\quad$ Solid tumor & $15(93.8)$ \\
HSCT & $1(6.2)$ \\
Inpatient unit [n (\%)] & $3,5(1-17.8)$ \\
$\quad$ Hematology/oncology unit & $10(62.5)$ \\
$\quad$ ICU & $2(12.5)$ \\
Lenght of stay until CRBSI [days (IQR)] & $14(87.5)$ \\
Catheter type [n (\%)] & $150(20-204)$
\end{tabular}

$\leq 500 / \mathrm{mm}^{3}$ within the period of 3 days before and after the culture day.

IQR: Interquertile range, HSCT: hematopoietic stem cell transplantation, ICU: intensive care unit, CRBSI: catheter-related bloodstreaminfection, ANC: absolute neutrophil count. 
Table II. Microorganisms in catheter-related bloodstream infections.

\begin{tabular}{lcr}
\hline Organisms [n (\%)] & & \\
\hline Gram positive & $8(50)$ & \\
$\quad$ Coagulase-negative staphylococci & $5(31.3)$ & \\
Gram negative & & $1(6.2)$ \\
$\quad$ Klebsiella oxytoca & & $1(6.2)$ \\
$\quad$ Pseudomonas aeruginosa & & $1(6.2)$ \\
$\quad$ Escherichia coli & & $1(6.2)$ \\
$\quad$ Acinetobacter baumannii & $2(12.5)$ & $1(6.2)$ \\
$\quad$ Enterobacter cloacae & & $1(6.2)$ \\
Polymicrobial & & $1(6.2)$ \\
$\quad$ Stenotrophomonas maltophila, Bacillus cereus & $1(6.2)$ & \\
$\quad$ Acinetobacter lwoffii, Enterococcus faecium & & $1(6.2)$ \\
Fungus & & \\
$\quad$ Candida pelliculusa & & \\
\hline
\end{tabular}

the guideline of Infectious Diseases Society of America: vancomycin $5 \mathrm{mg} / \mathrm{ml}$, ceftazidime $0.5 \mathrm{mg} / \mathrm{ml}$ and ciprofloxacin $0.2 \mathrm{mg} / \mathrm{ml} .^{5}$ caspofungin -the only antifungal lock- was administered as $10 \mathrm{mg}$ caspofungin with 200 IU heparin in a $3 \mathrm{ml}$ solution based on former experience in the literature. ${ }^{10}$ Dwell time was 24 hours for all but one in which the patient received 12-hour-vancomycin and 12-hourceftazidime lock. Time interval from the first positive culture to ALT was 2.5 (IQR: 2-3,7) days. In terms of complications, bleeding developed in two cases and occlusion in only one patient. Bleeding was not severe in both events but the occluded catheter had to be removed within 14 days after the occlusion. Heparin concentration in lock solutions was $100 \mathrm{IU} / \mathrm{ml}$ in both patients with bleeding but they were two and four months old. Occlusion occured in a 7 month-old patient who had a lock solution containing 2500 IU/ml heparin (Table III).

Frequency of ALT failure was 31.2\% (5/16) in the present study. When successful and unsuccessful ALT outcome groups were compared with each other, a statistically significant difference was observed only for age (Table IV). Median age of unsuccessful group was notably lower than the successful group ( 0.9 vs 6.82 years. $p=0.038$ ). The catheter was removed from two patients due to catheter dysfunction within 14 days in the first case and due to persistant clinical findings such as fever despite 72 hours of appropriate therapy in the second. Other reasons for treatment failure were recurrences in two cases and re-infection in the other one within 30 days after discontinuation of ALT. The pathogens in the unsuccessful group were coagulase negative staphylococci (CoNS) in three, Enterobacter cloacae in one and polimicrobial agents (Acinetobacter lwoffi and Enterococcus faecium) in the last.

All patients were followed up for recurrence or re-infection until catheter removal, death or the end of the study period. Median of surveillance period was 9.2 months (2.5-16.3) in the successful treatment group and 3.6 months (1.1-12.8) in all cases. Recurrence was observed in about one-fourth of the patients and reinfection occurred in $16.7 \%$ during surveillance period. On the 30th day, all children included in the study were alive. Causative agents in the first and second episodes of both recurrences and reinfections were all CoNS except for one in whom E. cloacae was isolated in the first episode but reinfection was observed with a CoNS again. With an additional statistical analysis we found younger age to be a significant risk factor not only for treatment failure but also for recurrence and/or reinfection. The median age was 0.89 years in patients with recurrence or reinfection and 11.4 years in others $(p=0.011)$. 
Table III. Characteristics of antibiotic lock therapies.

\begin{tabular}{llrl}
\hline & & $\mathrm{n}$ & $(\%)$ \\
\hline Antibiotic locks & Vancomycin $(5 \mathrm{mg} / \mathrm{ml})$ & 8 & $(50)$ \\
& Ceftazidime $(0.5 \mathrm{mg} / \mathrm{ml})$ & 3 & $(18.6)$ \\
& Ciprofloxacin $(0.2 \mathrm{mg} / \mathrm{ml})$ & 2 & $(12.5)$ \\
& Caspofungin $(3.3 \mathrm{mg} / \mathrm{ml})$ & 1 & $(6.3)$ \\
& Vancomycin $(5 \mathrm{mg} / \mathrm{ml})$-Ciprofloxacin $(0.2 \mathrm{mg} / \mathrm{ml})^{*}$ & 1 & $(6.3)$ \\
& Vancomycin $(5 \mathrm{mg} / \mathrm{ml})$-Ceftazidime $(0.5 \mathrm{mg} / \mathrm{ml})^{* *}$ & 1 & $(6.3)$ \\
Heparin concentration $(\mathrm{IU} / \mathrm{mL})$ & 7 & $(46.7)$ \\
& 100 & 3 & $(20.0)$ \\
& 2500 & 5 & $(33.3)$ \\
& 5000 & Median & $($ Range $)$ \\
Others & & 24 & $(12-24)$ \\
& Dwell time, h & 10,5 & $(7-17)$ \\
& Duration, $\mathrm{d}$ & 2,5 & $(1-8)$ \\
& Time to ALT, d & $\mathrm{n}$ & $(\%)$ \\
& & 1 & $(6.3)$ \\
& Occlusion & 2 & $(12.5)$ \\
\hline
\end{tabular}

${ }^{*}$ consecutively.

**simultaneous.

$\mathrm{H}$ : hour, d: days, Time to ALT: number of days from collection of first positive blood culter to initiation of antibiotic lock therapy, TPA: tissue plasminogen activator.

\section{Discussion}

The most common adverse effects of central venous access devices are catheter-related bloodstream infections. ${ }^{11}$ According to the 2017 summary report of the national health servicerelated infections surveillance network, the overall CRBSI rate in all pediatric intensive care units in our country is $4.8 / 1000$ catheterdays and 5.6 in pediatric intensive care units in university hospitals. ${ }^{12}$ CRBSI rate in a single pediatric hematology-oncology unit in Turkey was found to be 7.4/1000 catheter-days. ${ }^{8}$ In our research CRBSI rate was calculated in patients who underwent ALT and found to be 5.64/1000 catheter-days. Our results are consistent with the data in our country.

The most common infectious agents in surgically inserted catheters are known to be coagulase negative staphylococci, followed by enteric gram negative bacilli, $S$. aureus and P. aeruginosa..$^{5}$ In a study by Wolf et al.; grampositive, gram-negative, polymicrobial agents and Candida species were found to be the most prevelant cause of CRBSIs among 94 pediatric cancer patients. Our data are similar to previous studies in the pediatric patient group and especially in childhood malignancies in terms of the distribution of CRBSIs agents. ${ }^{8,13-15}$

When CLABSI is related to a long term CVC catheter salvage can be attempted by administering both systemic antibiotics and antibiotic lock according to current guidelines. Yet, catheter salvage is not recommended in the following circumstances: complicated CRBSI (suppurative thrombophlebitis, endocarditis, osteomyelitis, or other metastatic infection), severe sepsis or hemodynamic instability, persistent bacteremia despite 72 hours of antibiotic therapy to which the infecting organism is susceptible, infections caused by Staphylococcus aureus, Pseudomonas aeruginosa, fungi, mycobacteria or Bacillus species and in case of tunnel infections, port abscesses or exit site infections. ${ }^{5}$ In our study, antibiotic lock therapies were administered with respect to recommendations of the latest guidelines. However, some conditions that ALT is not 
Table IV. Comparison of patient groups with antibiotic lock therapy success and failure.

\begin{tabular}{lrlrlc}
\hline & Success $(\mathrm{n}=11)$ & \multicolumn{1}{c}{ Failure $(\mathrm{n}=5)$} & $p$ \\
\hline Age, y (IQR) & 6.82 & $(1.2-12.2)$ & 0.9 & $(0.5-2.3)$ & 0.038 \\
Gender [n (\%)] & & & & & 0.596 \\
$\quad$ Male & 4 & $(36.4)$ & 3 & $(60)$ & \\
$\quad$ Female & 7 & $(63.6)$ & 2 & $(40)$ & \\
Underlying disease [n (\%)] & & & & & 0.596 \\
$\quad$ Leukemia & 7 & $(63.6)$ & 2 & $(40)$ & \\
$\quad$ Solid tumor & 4 & $(36.4)$ & 3 & $(60)$ & \\
Allogenic HSCT [n (\%)] & 1 & $(9.1)$ & 1 & $(20)$ & $>0.99$ \\
ANC, /mm ${ }^{3}$ (IQR) & 300 & $(0-1325)$ & 500 & $(0-2400)$ & 0.953 \\
Neutropenia [n (\%)] & 6 & $(54.5)$ & 4 & $(80)$ & 0.580 \\
Duration of neutropenia, d (IQR) & 1 & $(1-9.5)$ & 3.5 & $(1.3-23.8)$ & 0.476 \\
Lymphocyte count, /mm ${ }^{3}$ (IQR) & 300 & $(300-1150)$ & 1250 & $(375-1600)$ & 0.503 \\
Monocyte count, /mm ${ }^{3}$ (IQR) & 100 & $(0-250)$ & 200 & $(200-)$ & 0.209 \\
CRP, mg/L (IQR) & 36 & $(7-69)$ & 27 & $(11.5-70)$ & $>0.99$ \\
LOS until CRBSI, d (IQR) & 3 & $(1-10)$ & 11 & $(1.5-46.5)$ & 0.320 \\
Catheter-day, (IQR) & 141 & $(18-196)$ & 168 & $(31-307)$ & 0.743 \\
Catheter type [n (\%)] & & & & & $>0.99$ \\
$\quad$ Tunneled double-lumen catheter & 1 & $(9.1)$ & 4 & $(20)$ & \\
$\quad$ Port catheter & 10 & $(90.9)$ & 3 & $(80)$ & \\
Time to ALT, d (IQR) & 2 & $(1-3)$ & 2500 & $(100-3750)$ & 0.953 \\
Heparin concentration, IU/ml (IQR) & 1300 & $(100-5000)$ & 24 & $(18-24)$ & 0.583 \\
Dwell time, h (IQR) & 24 & $(24-24)$ & & \\
\hline
\end{tabular}

y: year, IQR: interquertile range, HSCT: hematopoietic stem cell transplantation, ANC: absolute neutrophil count, d: day, CRP: C-reactive protein, LOS: length of stay, CRBSI: catheter-related blood stream infection, ALT: antibiotic lock therapy, h: hour.

recommended but yet performed can come to one's attention. These were the cases who have really high risks concerning the complications of the catheter removal procedure and a crutial need for intravenous access. Candida pelluculosa, $P$. aeruginosa and polimicrobial agents involving Bacillus spp. were isolated from those three patients. Treatment was carried out with close monitoring of clinical and laboratory findings and eventually, ALT outcome was favourable in all. After the publication of recent guidelines, there have been many research findings indicating that ALT may also be beneficial for the pathogens mentioned above. ${ }^{16-18}$

ALT has its own risks. The potential for occlusion or systemic exposure of anticoagulants and antibiotics exists. We observed occlusion in one $(6.3 \%)$ and bleeding in two $(12.5 \%)$ patients.
However, it is not possible to attribute those to ALT since there was no control group. In the study by Wolf et al., ${ }^{19}$ there were no difference regarding ALT complications between the two groups, "ALT plus systemic antibiotic" and "systemic antibiotics only".

There have been different definitions and results in the literature concerning the success and effectiveness of antibiotic lock therapy. In a single randomized placebo-controlled trial, treatment failure was found to be $33 \%$ with adjunctive ALT (and 57\% in systemic treatment only, $\mathrm{p}=0.10$ ) on day $180 .{ }^{20}$ However, this trial does not reflect our target population as it was performed among 44 CRBSI episodes including various populations such as children, adults, hematology/oncology, hemodialysis and gastroenterology patients. In another study, 
success rate of ALT in adult cancer patients was reported to be $75.9 \%$ and was not very different from our results. ${ }^{21}$ In our study the failure rate of lock therapy was $31.2 \%$. Similar results despite different populations are also noteworthy.

In a case control study conducted by Wolf et al., ${ }^{19}$ to determine the benefit of ALT in pediatric cancer patients, failure rate was not different statistically in "systemic antibiotic plus ALT" $(50 \%)$ and "systemic therapy alone" groups $(38.4 \%, \mathrm{p}=0.24)$. Treatment failure was defined as catheter withdrawal due to infection within 14 days of the CRBSI onset, death due to infection or relapse with the same microorganism within 252 days. The fact that our failure rate is relatively low compared to Wolf et al. can be explained by differences in definitions such as the time period for relapse.

Factors for ALT failure have been investigated poorly in the literature. In a study within pediatric hemodialysis patients by Onder et al. ${ }^{22}$ younger age and higher serum phosphorus levels were associated with both treatment failure and reinfection. In a study conducted by Chen et al. ${ }^{23}$ in pediatric cancer patients whose catheter was removed due to CRBSI, catheter withdrawal was observed in $11.9 \%$, and the majority ( 9 of 17 patients, $52 \%$ ) were under two years of age. When we evaluated the factors leading to failure in ALT (which was observed as $31.2 \%$ ), the main risk factor was found to be younger age alone (0.9 vs 6.8 years, $\mathrm{p}=0.038$ ). Despite the differences in definitions and research groups our data are consistent with the existing literature.

In a systematic review and meta-analysis of various patient groups on anti-infective lock therapies, $20 \%$ relapse was reported in patients receiving ALT. $^{24}$ Adler et al. ${ }^{9}$ observed that $45(51 \%)$ of 87 catheters rescued in children with cancer had reinfection or recurrence. The pathogen was CoNS in all but one of the 12 patients with recurrence, and multivariate analysis revealed previous CRBSI due to CoNS and older age as significant risk factors for recurrence. Of note, the recurrence and reinfection rates were $23.1 \%$ and $16.7 \%$ in our study, and all recurrences and reinfections were also with CoNS. Results of our study was similar to Adler's in the standpoint of pathogens of recurrences or reinfections. In contrast to Adler's, we found younger age to be a significant risk factor for recurrence or reinfection (median 0.89 vs 11.4 years, $\mathrm{p}=0.011$ ).

The current study has some limitations. First of all, the sample size is not sufficiently large to perform multivariate risk factor analysis. Secondly, its retrospective and single-center design could allow confounders to affect the results and cannot be generalized to all populations. We believe that our center's experience contributes to the literature in spite of its limitations, as it is the first report which evaluates ALT outcome in pediatric cancer patiens from Turkey.

Although recommended in the last guidelines for diagnosis of CRBSIs, differencial time to positivity and quantitative blood cultures could not be used in the present study, as these techniques have recently been applied in laboratories of our hospital. But, we consider this not to be a serious limitation. Firstly, many previous clinical studies did not use those criteria in a similar way. ${ }^{6,19,25}$ Secondly, in a study by Wolf et al, ${ }^{19}$ analysis of episodes meeting the more stringent definition of "proven CRBSI" using differencial time to positivity showed similar rates of treatment failure.

Antibiotic lock therapy was found to be a safe modality with a success rate of $68.8 \%$ in pediatric cancer patients at our center and younger age was an independent risk factor for treatment failure. Future studies with larger sample sizes are needed to determine the factors affecting the ALT outcome, especially in childhood cancers. Identifying groups with low treatment success may lead to changes in the population in which ALT was recommended and may increase treatment success. Our study is important since it is the first report which evaluates ALT outcome in pediatric hematology/oncology patients from Turkey. 


\section{REFERENCES}

1. Mirro J Jr, Rao BN, Stokes DC, et al. A prospective study of Hickman/Broviac catheters and implantable ports in pediatric oncology patients. J Clin Oncol 1989; 7: 214-222.

2. Wilson MZ, Rafferty C, Deeter D, Comito MA, Hollenbeak CS. Attributable costs of central lineassociated bloodstream infections in a pediatric hematology/oncology population. Am J Infect Control 2014; 42: 1157-1160.

3. Wolf J, Connell TG, Allison KJ, et al. Treatment and secondary prophylaxis with ethanol lock therapy for central line-associated bloodstream infection in paediatric cancer: a randomised, double-blind, controlled trial. Lancet Infect Dis 2018; 18: 854-863.

4. Karagiannidou S, Zaoutis T, Maniadakis N, Papaevangelou V, Kourlaba G. Attributable length of stay and cost for pediatric and neonatal central line-associated bloodstream infections in Greece. J Infect Public Health 2019; 12: 372-379.

5. Mermel LA, Allon M, Bouza E, et al. Clinical practice guidelines for the diagnosis and management of intravascular catheter-related infection: 2009 Update by the Infectious Diseases Society of America. Clin Infect Dis 2009; 49: 1-45.

6. Schoot RA, van Dalen EC, van Ommen $\mathrm{CH}$, van de Wetering MD. Antibiotic and other lock treatments for tunnelled central venous catheterrelated infections in children with cancer. Cochrane Database Syst Rev 2013; 25:CD008975.

7. Norris LB, Kablaoui F, Brilhart MK, Bookstaver PB. Systematic review of antimicrobial lock therapy for prevention of central-line-associated bloodstream infections in adult and pediatric cancer patients. Int J Antimicrob Agents 2017; 50: 308-317.

8. Celebi S, Sezgin ME, Cakır D, et al. Catheterassociated bloodstream infections in pediatric hematology-oncology patients. Pediatr Hematol Oncol 2013; 30: 187-194.

9. Adler A, Yaniv I, Solter E, et al. Catheter-associated bloodstream infections in pediatric hematologyoncology patients: factors associated with catheter removal and recurrence. J Pediatr Hematol Oncol 2006; 28: 23-28.

10. Özdemir H, Karbuz A, Çiftçi E, et al. Successful treatment of central venous catheter infection due to Candida lipolytica by caspofungin-lock therapy. Mycoses 2011; 54: e647-e649.

11. Beck O, Muensterer O, Hofmann S, et al. Central venous access devices (CVAD) in pediatric oncology patients-a single-center retrospective study over more than 9 years. Front Pediatr 2019; 7: 260.
12. Hekimoğlu C, Batır E. Ulusal Sağlık Hizmeti İlişkili Enfeksiyonlar Sürveyans Ağ1 Özet Raporu 2017. Ankara, 2018.

13. Chaudhary M, Bilal MF, Du W, Chu R, Rajpurkar M, McGrath EJ. The impact of ethanol lock therapy on length of stay and catheter salvage in pediatric catheter-associated bloodstream infection. Clin Pediatr (Phila) 2014; 53: 1069-1076.

14. Aygün F, Aygün D, Çokuğraş H, Çam H, Camcıoğlu Y. Çocuk hastalarda kateter ilişkili enfeksiyon sıklığı; bir yıllık deneyim. Çocuk Enfeksiyon Dergisi 2017; 11: 76-81.

15. Pektaş A, Kara A, Gurgey A. Cohort study: Central venous catheter-related complications in children with hematologic diseases at a single center. Turkish J Hematol 2015; 32: 144-151.

16. Del Pozo JL, Alonso M, Serrera A, Hernaez S, Aguinaga A, Leiva J. Effectiveness of the antibiotic lock therapy for the treatment of port-related enterococci, Gram-negative, or Gram-positive bacilli bloodstream infections. Diagn Microbiol Infect Dis 2009; 63: 208-212.

17. Nucci M, Anaissie E, Betts RF, et al. Early removal of central venous catheter in patients with candidemia does not improve outcome: analysis of 842 patients from 2 randomized clinical trials. Clin Infect Dis 2010; 51: 295-303.

18. Funalleras G, Fernandez-Hidalgo N, Borrego A, et al. Effectiveness of antibiotic-lock therapy for long-term catheter-related bacteremia due to gramnegative bacilli: a prospective observational study. Clin Infect Dis 2011; 53: e129-e132.

19. Wolf J, Allison KJ, Tang L, Sun Y, Hayden RT, Flynn PM. No evidence of benefit from antibiotic lock therapy in pediatric oncology patients with central line-related bloodstream infection: results of a retrospective matched cohort study and review of the literature. Pediatr Blood Cancer 2014; 61: 18111815.

20. Rijnders BJ, Van Wijngaerden E, Vandecasteele SJ, Stas M, Peetermans WE. Treatment of long-term intravascular catheter-related bacteraemia with antibiotic lock: randomized, placebo-controlled trial. J Antimicrob Chemother 2005; 55: 90-94.

21. Freire MP, Pierrotti LC, Zerati AE, et al. Role of lock therapy for long-term catheter-related infections by multidrug-resistant bacteria. Antimicrob Agents Chemother 2018; 62: e00569-18.

22. Onder AM, Billings A, Chandar J, et al. PREFABL: Predictors of failure of antibiotic locks for the treatment of catheter-related bacteraemia. Nephrol Dial Transplant 2010; 25: 3686-3693. 
23. Chen SH, Yang CP, Jaing TH, Lai JY, Hung IJ. Catheter-related bloodstream infection with removal of catheter in pediatric oncology patients: a 10-year experience in Taiwan. Int J Clin Oncol 2012; 17: 124-130.

24. O'Horo JC, Silva GLM, Safdar N. Anti-infective locks for treatment of central line-associated bloodstream infection: a systematic review and meta-analysis. Am J Nephrol 2011; 34: 415-422.
25. Megged O, Shalit I, Yaniv I, Fisher S, Livni G, Levy I. Outcome of antibiotic lock technique for persistent central venous catheter-associated coagulasenegative Staphylococcus bacteremia in children. Eur J Clin Microbiol Infect Dis 2010; 29: 157-161. 